\title{
Smoking and its association with serum lipid levels
}

\author{
Singh D.P. ${ }^{1}$, Gulati D. ${ }^{2}$, Singh P. $^{3}$ \\ ${ }^{1}$ Dr. Devendra Pratap Singh Rajput, Associate Professor, Medicine, L. N. Medical College, Bhopal, ${ }^{2}$ Dr. Divyani Gulati, \\ M.B.B.S. Intern, L.N. Medical College, Bhopal, ${ }^{3}$ Dr. Priti Singh, Assistant Professor, Ophthalmology, Gandhi Medical \\ College, Bhopal, MP, India.
}

Address for Correspondence: Dr. Devendra Pratap Singh Rajput, Associate Professor, Medicine, L.N. Medical College, Bhopal, A-4, Staff Quarters, J. K. Hospital Campus, Kolar Road, Bhopal (Madhya Pradesh). Email-devprasin20@gmail.com

\begin{abstract}
Objective: To compare the effect of smoking over lipid profile. Method: Total 100 subjects were included in this study, 50 were smoker and 50 non-smoker. All the factors other then smoking those can lead to dyslipidemia were ruled out. All patient's fasting blood sample was collected and lipid profile was estimated. Data was analysed and 't' value was calculated. Results: Serum levels of total cholesterol (TC), triglycerides (TG), low density cholesterol (LDL), very low density cholesterol (VLDL) were found to be significantly high in smokers in comparison to non-smokers but high density cholesterol (HDL) did not any significant difference between two groups. Conclusion: Smoking affects lipid profile significantly, number and duration of smoking having correlation with serum lipoprotein levels but type of smoking (cigarette or biddi) doesn't change the outcome.
\end{abstract}

Key word- Smoking, Total cholesterol, LDL, VLDL, Triglyceride, HDL

\section{Introduction}

Smoking is one of the major cause of mortality and morbidity throughout the world. And as per the reports of World Health Organisation, India is home for $12 \%$ of the world's smokers and approximately 900,000 people die every year in India due to smoking as of 2009 [1].

Smokers have a higher risk of coronary artery disease than non-smokers, various explanation have been offered for its association, including altered blood coagulation [2] impaired integrity of arterial wall [3] and changes in blood lipid and lipoprotein leading to increase in concentration of total cholesterol, LDLcholesterol, VLDL-cholesterol, triglyceride, and fall in level of anti-atherogenic HDL-cholesterol, as per the reports of various workers [4-8].

Dyslipidemia, as a risk factor for cardiovascular diseases, is manifested by elevation or attenuation of plasma concentration of lipoproteins [9]. It is presence of abnormal level of lipids in blood characterised by

Manuscript received $24^{\text {th }}$ October 2016

Reviewed: $6^{\text {th }}$ November 2016

Author Corrected: $17^{\text {th }}$ November 2016

Accepted for Publication 30 $0^{\text {th }}$ November 2016 elevation of concentration of total cholesterol, LDL (low density lipoprotein), TG (triglyceride) and decrease in concentration of HDL (high density lipoprotein) [10].

A comprehensive meta-analysis by Craig et al. examined published data from 1966 to 1987 and estimated the risk caused by smoking on CVD with particular emphasis on lipid and lipoprotein involvement [11]. Various mechanisms leading to lipid alteration by smoking are:

(a) Nicotine stimulates sympathetic adrenal system leading to increased secretion of catecholamine's resulting in increased lipolysis and increased concentration of plasma free fatty acids (FFA) which further results in increased secretion of hepatic FFAs and hepatic TG along with VLDL-C in blood stream [8,12]. (b) Fall in oestrogen levels occur due to smoking which further leads to decreased HDL-cholesterol[13] (c) presence of hyperinsulinemia in smokers leads to increased cholesterol, LDL-C, VLDL-C and TG due to decreased activity of lipoprotein lipase [14-15]. 
(d) Consumption of diet rich in fat and cholesterol as well as diet low in fibre and cereals content by smokers as compared to non-smokers $[8,16]$.

Thus a strong synergistic interaction exist between hypercholesterolemia and smoking in genesis of various vascular complications, clearing the relationship might be important for increasing the proportion of longevity in population.

\section{Material and Method}

The aims of our study are:

1. To study alteration in lipid profile in healthy smokers and compare the same with lipid profile of non smokers

2. To find out dose response correlation between the numbers of cigarette/biddi smoked to the degree of alteration in lipid profile

3. To study lipid profile alteration with duration of smoking.

Study Design: Comparative cross sectional study.

Study Population: subjects taken from the healthy attendants of OPD patient visiting our hospital. Study population taken from age 17-60 years. It was divided into control and study group. Subjects selected for the study were 50 healthy smokers and 50 healthy nonsmokers. Our population was included male subjects because in our society male smoke frequently and openly as compared to female, and female if do smoke are reluctant in admitting it.

Sample Size: 100 subjects were taken.

Selection Criteria (on the basis of history, general examination, and systemic examination).

\section{Observation and Results}

The study included 100 male subjects who were classified into smokers and non-smokers. The BMI for all the subject was determined and the difference for BMI and age was statistically insignificant ( $p$ value $=1.000$ )

Duration of smoking is around 17 yrs (16.98) and amount is around 15/day (14.8).

Table-1: Showing distribution of number of smokers and non-smokers in various age group.

\begin{tabular}{|c|c|c|}
\hline Age-group & Smokers(n1) & Non-smokers(n2) \\
\hline $16-25$ yrs & 5 & 3 \\
\hline $26-35 y r s$ & 8 & 14 \\
\hline $36-45 y r s$ & 17 & 14 \\
\hline $46-55 y r s$ & 16 & 4 \\
\hline$>55 y r s$ & 4 & 50 \\
\hline Total & 50 & 14 \\
\hline
\end{tabular}

*Most of the smokers were of age group 36-45yrs.
Inclusion Criteria- Healthy smokers and healthy nonsmokers (age 17-60yrs, BMI <30) were included. Subjects with no family history of dyslipidemia were included.

Exclusion Criteria- Subjects with diabetes, hypertension, renal disease, hepatic impairment, hypothyroidism and obesity were excluded. Subjects with history of alcohol abuse and ex-smokers were excluded. Subjects taking any drug altering lipid profile (lipid lowering drugs, beta-blockers, glucocorticoids, oestrogen, progesterone and thiazide diuretics) were excluded.

Data Collection and Procedure: Written consent was taken from subjects. The purpose of study was explained to patients and assurance was given to them that procedure is harmless. Information on smoking habits was obtained using the questionnaires designed according to previous studies. General and systemic examination was done.Study was conducted in medicine department of the institute.

Permission for research was taken from institutional ethical committee after approval of research protocol. Identity of person is kept confidential. Blood samples were collected after an overnight fast for measurement of serum lipids (TG,LDL,HDL,VLDL,TC)

Cholesterol and triglyceride was measured by standard laboratory techniques using commercially available enzymatic kits (Roche diagnostic, COBAS c 111). Tests were performed by technician in biochemistry laboratory of JK hospital. SPSS software was used for statistical analysis. 
Table-2: Showing lipid profile in smokers and non-smokers.

\begin{tabular}{|c|c|c|c|}
\hline Lipids & $\begin{array}{c}\text { Smoker } \\
(\text { mean } \pm \text { 2 SD) }\end{array}$ & $\begin{array}{c}\text { Non-smoker } \\
(\text { mean } \pm 2 ~ S D)\end{array}$ & P-value $^{\#}$ \\
\hline TG & $135.98 \pm 60.12$ & $101.98 \pm 62.74$ & $<0.0001^{* *}$ \\
\hline LDL & $122.14 \pm 62$ & $100.06 \pm 58.55$ & $0.0004^{*}$ \\
\hline HDL & $38.94 \pm 33.70$ & $39.66 \pm 18.58$ & 0.7919 \\
\hline VLDL & $25.64 \pm 15.79$ & $21.18 \pm 12.33$ & $0.0022^{* *}$ \\
\hline TC & $184.98 \pm 62.60$ & $159.88 \pm 65.78$ & $0.0002^{* *}$ \\
\hline
\end{tabular}

**highly significant *significant \# ' $\mathrm{t}$ ' test for 2 samples.

Above table shows significant increase in value of TG, LDL-C, VLDL-C, and TC in smokers as compared to nonsmokers, while decrease in HDL-C values in smokers is statistically insignificant.

Table-3: Showing BMI of smokers and non-smokers.

\begin{tabular}{|c|c|c|c|}
\hline & Smokers (mean \pm 2 SD) & Non-smokers(mean $\pm 2 S D)$ & P-value \\
\hline BMI & $23.85 \pm 4.86$ & $23.85 \pm 5.34$ & 1 \\
\hline
\end{tabular}

Table-4: Correlation of duration of smoking with lipid value as per Pearson's correlation score(r)

\begin{tabular}{|c|c|c|}
\hline Lipid & Duration(r) & P value \\
\hline TG & 0.391 & 0.005 \\
\hline LDL & 0.478 & $<0.001$ \\
\hline HDL & -0.432 & 0.002 \\
\hline VLDL & 0.303 & 0.032 \\
\hline TC & 0.428 & 0.002 \\
\hline
\end{tabular}

Pearson's correlation coefficient shows less correlation between duration of smoking and rise in lipid values i.e. TC TG LDL VLDL and fall in level of HDL.

Table-5: Correlation of number of biddi/cigarette with lipid value as per Pearson's correlation score(r)

\begin{tabular}{|c|c|c|}
\hline Lipid & Number (r) & P value \\
\hline TG & 0.345 & 0.014 \\
\hline LDL & 0.273 & 0.055 \\
\hline HDL & -0.281 & 0.048 \\
\hline VLDL & 0.275 & 0.053 \\
\hline TC & 0.248 & 0.083 \\
\hline
\end{tabular}

Pearson's correlation coefficient shows very less correlation with number of cigarette/biddi smoked per day to alteration in lipid value.

Hence with above data we can say that duration and numbers of cigarette/biddi smoking having little correlation with alteration of lipid values but values are more significant for duration of smoking when we compare with number of cigarette/biddi smoked per day. 


\section{Discussion}

Our study revealed that smoking causes significant increase in concentration of triglyceride, TC, LDL-C and VLDL-C in smokers as compared to non-smokers. While the alteration in levels of HDL-C was not statistically significant $(\mathrm{p}$ value $=0.7919$ ) among the two groups. Mean serum total cholesterol was $184.98 \pm$ 62.60 in smokers as compared to that in non-smokers where the value was $159.88 \pm 65.78$, hence it is significantly raised $(\mathrm{p}=0.0002)$ which is similar to the findings observed in other studies.[11,17, 19,21,22,23].

Smoking also increases TG the value of which is significantly raised in smokers i.e. $135.98 \pm 60.12$ as compared to non-smokers where the value is $101.98 \pm$ 62.74 which is statistically very significant $(\mathrm{p}<0.0001)$ $[17,19,21]$. The trigly ceride / high-density lipoprotein abnormalities have recently been suggested to be related to insulin resistance. In fact, it has been proposed that insulin resistance is a potential key link between cigarette Smoking and cardiovascular disease [25]. Similarly values are also raised for LDL that is $122.14 \pm$ 62.00 in smokers as compared to $100.06 \pm 58.55$ in nonsmokers and VLDL which is $25.64 \pm 15.78$ in smokers as compared to $21.18 \pm 12.33$ in non-smokers, which is supported by studies done by Craig et al., Anila Jaleel et al.,NS Neki, Naisargi Joshi et al. Cheryl S Brischetto et al., D. J. Freeman et al. and Sinha A K et al [11, 17, 19, 21, 22, 23, 26] But Gepher AD et al., Dirican $M$ et al and Nesje LA et al haven't seen significant rise in LDL values in smokers and non-smokers [20, 27, 28] while Zhang Yan-Ling et al. in their study over residents of age group 90yrs or more have experienced decrease in value of LDL, TC, TG, and VLDL in smokers as compared to non-smokers [18].

One study showed slightly lower mean triglyceride levels in smokers as compared to that of non-smokers, but this study was conducted in young population of 2025 years of age group those who used to smoke for 5-7 cigarette per day and duration of smoking was only 5 year. [24]. Cigarette smoking also increases oxidative modification of LDL. Circulating products of lipid peroxidation and autoantibody titers to oxidized LDL are significantly increased in smokers [29]. In 1988, Yakode et al [30] reported that exposure to cigarette smoking caused a modification of LDL, which was actively taken up by the macrophages to form foamcells in culture. Frei et al [31] observed that exposure of human plasma to the gas phase of cigarette smoke caused oxidative modification of plasma LDL. Smoking associated with vasomotor dysfunction, inflammation, and modification of lipids which are integral components for the initiation and progression of atherosclerosis. These components precede the apparent structural and clinicopathologic manifestations of atherosclerosis [32, 33].

Although several studies [34, 35] provide the evidence that tobacco is strongly associated with altering the normal status of the lipid profile, there still is inconclusive evidence regarding the alteration of a particular lipoprotein, particularly to high density lipoprotein (HDL) levels. Some authors have concluded that HDL levels were same for smokers and nonsmokers [36], while others have found conflicting results wherein significant variations (low levels of HDL in cigarette smokers) were obtained [37, 38]. Our study doesn't show significant alteration in the values of HDL-C in smokers as well as non-smokers ( $\mathrm{p}=$ 0.7919 ) while different studies revealed that absenters had shown increase in HDL, total HDL and large HDL particles compared with those continuing smoking which is seen to be raised in non-smokers. While other studies shown a decrement in values in smokers $[17,18$, 19, 20, 21, 22, 23]. In another study conducted by Majos O. D. et al. [39] reported that there is significant decrease in HDL-C, but there is no change in total cholesterol and triglycerides. Another report shows lower but no significant HDL levels in smokers [40].

But a study conducted by Siekmeier et al [41] reported the HDL levels are same for smokers and non-smokers.

Here we also proved that alteration of lipid values shows correlation with duration and amount of cigarette/biddi smoked but values are more significant for duration of smoking as compared to number of cigarette/biddi smoked per day and studies by Craig et al. and Naisargi Joshi et al also showed correlation with the number of cigarette/biddi smoked [11, 21]. While studies conducted by Suleyman $\mathrm{H}$ et al. And Khurana $M$ et al. Showed that change in serum lipids tends to be high with the increase in duration and intensity both [42, 43].

The study by Gepher AD et al showed effects stronger in women, while our study mainly focused on males. [20]. 
This study was conducted primarily to study the effect of smoking over lipid profile and as this study has shown significant alteration in lipid profile with smoking and dyslipidemia is associated with increased cardiovascular disorders so result of this study can be used to create awareness about ill effects of smoking and that will be helpful in decreasing cardiovascular morbidity and mortality [44].

There are some limitation of this study also as results cannot be generalized because of small sample size and same geographical distribution. In this study we did not include Apolipoprotein A1 and Apolipoprotein B in lipid profile.

Reporting of smoking habits was by subjects them self which sometimes may not be accurate. Further studies should be multicentic with large sample size and should cover wide geographical area.

\section{Conclusion}

Serum levels of TC, LDL, VLDL, TG were significantly raised in smokers as compared to non-smokers, while the value of HDL remained statistically unchanged in both the groups. Serum lipoprotein levels also showed correlation with number and duration of biddi/cigarette smoked, but results were more significant for duration than number.

\section{Acknowledgement}

We would like to cordially thank to all the subjects for participating in this study and to the department of Medicine and Biochemistry of L. N. Medical College \& J. K. Hospital \& Research Centre, Bhopal (M.P.), India for supporting this study and college authority for allowing to conduct the study.

Funding: Nil, Conflict of interest: None initiated, Permission from IRB: Yes

\section{References}

1. Prabhat Jha, Binu Jacob,Vandhana Gajalakshmi, Prakash C. Gupta, Neeraj Dhingra, Rajesh Kumar et al.A nationally representative case control study of smoking and death in India NEJM 2008; 358(11): 1137-47.

2. McGill HC Jr. Potential mechanism for augmentation of atherosclerosis and atherosclerotic disease by cigarette smoking. Prev Med 1979; 8(3): 390-403.
3. Topping DL. effects of tobacco smoke and its constituents on lipid carbohydrate metabolism. In: Clemens MJ, Ashwell M, eds. Biochemistry of cellular regulation. vol 2. Boca Raton, Florida: CRC Press, 1980: $165-83$.

4. Carlson LA, Bottiger LE, Ahfeldt PE. Risk factors for myocardial infarction in Stockholm prospective study: A 14 year follow up on focusing on the role of plasma triglyceride and cholesterol. Acta Med Scand 1979; 206(5): 315-60.

5. Ole D. Mjos MD. Lipid effect of smoking. AM heart J 1988; 115(1): 272-5.

6. Rustogi R, Shrivastava SS, Mehrotra TN, Singh VS, Gupta MK. Lipid profile in smokers. JAPI 1989; 37(12) 764-6.

7. Austin MA. Plasma triglyceride and coronary heart disease. Arterio Throm 1991; 11(1): 2-14.

8. Muscat JE, Harris RE, Haley JE, Wynder JE. Cigarette smoking and plasma cholesterol. AM Heart J 1991; 121(1): 141-7.

9. Hossein Fakhrzadeh, Ozra Tabatabaei-Malazy. Dyslipidemia and cardiovascular disease. ISBN 2012: 978-953-307-904-2.

10. Nelson Fausto, Vinay Kumar, jon c.Aster, Ann Abbas. Robbins and Cotran pathologic basis of disease. $8^{\text {th }}$ edition.2012:1144-1145.

11. Craig WY, Palomaki GE, HaddowJE: cigarette smoking and serum lipid and lipoprotein concentration: an analysis of published data.BMJ 1989; 298(6676): 784-8.

12. Simons LA, Simons J, Jones AS. The interaction of body weight, age, cigarette smoking and hormone usage with blood pressure and plasma lipids in an Australian community. Aus NZ Med 1984; 14(3): 215-21.

13. Benouwitz NL. Pharmacologic aspect of cigarette smoking and nicotine addiction. New Engl J Med 1998; 319(20): 1318-30.

14. Stalder M, Pometta B, Suenram A. Relationship between plasma insulin levels and HDL-Cholestrol in healthy men. Diabtologia 1981; 21(6): 544-8. 
15. Reaven GM. Role of insulin resistance in human disease. Diabetes. 1988; 37(12): 1595-1607.

16. Wynder EL, Hariss. Population screening for plasma cholesterol. Community based results from Connecticut. Am Heart J 1989; 117(3): 649-56.

17. Anila jaleel,Farhan Jaleel, Rahan Majeed and Ejaz Alam. Leptin and bllod lipidlevels in smokers and exsmokers. world applied sciences journal 2007; 2(4): 348-352.

18. Zhang Yan-Ling, Zhan Dong-Quing, Huang ChagQuan and Dong Bi-Rong. Cigarette smoking and its association with serum lipid/lipoprotein among Chinese nonagenarians/centenarians. Lipids in health and disease 2012;11(1):1-6.

19. NS Neki.Lipid profile in chronic smokers. JIACM 2002; 3(1): 51-4.

20. Gepner AD, Piper ME, Johnson HM, Fiore MC, Baker TB, Stein JH. Effects of smoking and smoking cessation on lipids and lipoproteins: outcomes from a randomized clinical trial. Am Heart J. 2011 Jan; 161 (1): 145-51. doi:10.1016/j.ahj.2010. 09. 023.

21. Naisarg Joshi, Chinmay Shah, HB Mehta, PA Gokhledone.Comparative study of lipid profile on healthy smokers and non-smokers. Int J Med Sci Public Health. 2013; 2(3): 622-626.

22. Cheryl S Brischetto PhD, William E. Connor MD, Sohja L. Connor MS, Joseph D. Matarazzo PhD. Plasma lipid and lipoprotein profiles of cigarette smokers from randomly selected families: Enhancement of hyperlipidemia and depression of HDL. Am J Cardiol 1983; 52(7): 675-80.

23. Freeman DJ, Graffin BA, Murray E, Lindsay GM, Gaffney D, Packard CJ, et al. smoking and plasma lipoprotein in man: effects on low density lipoprotein cholesterol levels and high density lipoprotein sub fraction distribution. Eur J Clin Invest. 1993;23(10) : 630-40.

24. Imran Khan, Mohd. Farhan, Sai Ramesh A, Padma Thiagarajan. Effects of smoking on serum lipid levels in nascent young Indian smokers. Int. J Pharma Sci And Research.2012;3(9):463-466.
25. Reaven G, Tsao P.S; Insulin resistance and compensatory hyperinsulinemia. The key player between cigarette smoking and cardio-vascular disease. J Am Coll Cardiol. 2003;41(6):1044-7.

26. Sinha AK, Misra GC, Patel DK. Effect of smoking on lipid profile in the young. J Assoc Physicians India 1995;43(3):185-8.

27. Dirican M , Sarandol E, Ulukaya E, Tokullugil HA. Effects of smoking on serum lipid and lipoprotein concentrations and lecithin: cholesterol acyltransferase activity. J Med Invest 1999; 46(3-4):169-72.

28. Nesje LA, Mjøs OD. Plasma HDLc holesterol and the subclasses HDL2 and HDL3 in smokers and nonsmokers. Artery 1985; 13(1):7-18.

29. Heitzer T, Yla-Herttuala S, Luoma J; Cigarette smoking potentiates endothelial dysfunction of forearm resistance vessels in patients with hypercholesterolemia. Role of oxidized LDL. Circulation. 1996;93(7):13461353.

30. Yokode M, Kita T, Arai H; Cholesteryl ester accumulation in macrophages incubated with low density lipoprotein pretreated with cigarette smoke extract. Proc Natl Acad Sci USA. 1988;85(7):2344-8.

31. Frei B, Forte T.M, Ames B.N, Cross C.E; Gas phase oxidants of cigarette smoke induce lipid peroxidation and changes in lipoprotein properties in human blood plasma. Protective effects of ascorbic acid. Biochem J. 277 1991;277(pt1)133-138.

32. Ross R; Atherosclerosis - an inflammatory disease. N Engl J Med.1999;340(24):115-26.

33.Clarkson T.B, Weingand K.W, Kaplan J.R; Mechanisms of atherogenesis. Circulation. 1987 Jul; 76 (1 Pt 2):I20-8.

34. Cuesta C, Sánchez-Muniz FJ, et al. Effect of age and cigarette smoking on serum concentrations of lipids and apolipoproteins in a male military population. Atherosclerosis 1989;80(1); 33-9.

35. Arsian E, Yakar T, Yavaşoğu I. The effect of smoking on mean platelet volume and lipid profile in young male subject. Anadolu Kardio Derg 2008; $8(6): 422-5$. 
36. Siekmeier R, März W, Kronenberger H, Seiffert UB, Gross W. Effects of cigarette smoking and plasma lipids, apolipoproteins and lipoprotein (a) in healthy subjects. Clin Chem 1994;40(7):1350-1.

37. Criqui MH, Wallace RB, Heiss G, et al. Cigarette smoking and plasma high density lipoprotein cholesterol.The lipid Research clinics Programme Prevalence study. Circulation, 1980;62(4):IV70-76.

38. Ito $\mathrm{T}$, Nishiwaki $\mathrm{M}$, Ishikawa $\mathrm{T}$, Nakamura $\mathrm{H}$. CETP and LCAT activities are unrelated to smoking and moderated alcohol consumption in healthy normolipidemic men. Jpn Circ J 1995; 59(8):541-6.

39. Mjøs OD. Lipid effects of smoking. Am Heart J. 1988 Jan;115(1 Pt 2):272-5.

40. Lopes PA, Santos MC, Vicente L, Viegas-Crespo AM. Effect of cigarette smoking on serum alpha- tocopherol and the lipid profile in a Portuguese population. Clin Chim Acta. 2004 Oct;348(1-2):49-55.

41. Siekmeier R, Wülfroth P, Wieland H, Gross W, März W. Low-density lipoprotein susceptibility to in vitro oxidation in healthy smokers and nonsmokers. Clin Chem. 1996 Apr;42(4):524-30.

42. Suleyman H, Gumustekin K, Taysi S, et al. Beneficial effects of Hippo phae rhamnoides $\mathrm{L}$ on nicotine induced oxidative stress in rat blood compared with vitamin E. Biol Pharm Bull2002;25(9):1133-6.

43. Khurana M, Sharma D, Khandelwal PD. Lipid profile in smokers and tobacco chewers - a comparative study. J Assoc Physicians India 2000;48(9):895-7.

44. Ezzati M, Henley SJ, Thun MJ, Lopez AD. Role of smoking in global and regional cardiovascular mortality. Circulation. 2005 Jul 26;112(4):489-97. Epub 2005 Jul 18.

\section{How to cite this article?}

Singh D.P., Gulati D., Singh P. Smoking and its association with serum lipid levels. Int J Med Res Rev 2016;4(11):20642070.doi:10.17511 /ijmrr. 2016.i11.28. 